\title{
BMJ Open Characteristics and quality of clinical practice guidelines addressing acupuncture interventions: a systematic survey of 133 guidelines and 433 acupuncture recommendations
}

Xiaorong Tang (D) , ${ }^{1}$ Xiaoshuang Shi (D) , ${ }^{2,3}$ Hong Zhao, ${ }^{3,4}$ Liming Lu, ${ }^{1}$ Ze Chen, ${ }^{1}$ Yixuan Feng, ${ }^{3}$ Lanping Liu, ${ }^{5}$ Ruihua Duan, ${ }^{6}$ Pingping Zhang, ${ }^{5}$ Yuqin $\mathrm{Xu},{ }^{3}$ Shuo Cui, ${ }^{3}$ Fen Gong, ${ }^{6}$ Jingwen Fei, ${ }^{5}$ Neng-Gui Xu, ${ }^{1}$ Xianghong Jing, ${ }^{3}$ Gordon Guyatt, ${ }^{7,8}$ Yu-Qing Zhang (D) 3,7,9,10

To cite: Tang X, Shi X, Zhao H, et al. Characteristics and quality of clinical practice guidelines addressing acupuncture interventions: a systematic survey of 133 guidelines and 433 acupuncture recommendations. BMJ Open 2022;12:e058834. doi:10.1136/ bmjopen-2021-058834

- Prepublication history and additional supplemental material for this paper are available online. To view these files, please visit the journal online (http://dx.doi.org/10.1136/ bmjopen-2021-058834).

$\mathrm{XT}$ and XS contributed equally.

Received 31 October 2021 Accepted 17 January 2022

Check for updates

(c) Author(s) (or their employer(s)) 2022. Re-use permitted under CC BY-NC. No commercial re-use. See rights and permissions. Published by BMJ.

For numbered affiliations see end of article.

Correspondence to Dr Yu-Qing Zhang; madisonz1220@gmail.com and Dr Xianghong Jing; jxhtjb@263.net

\section{ABSTRACT}

Objective To systematically summarise acupuncturerelated Clinical Practice Guidelines (CPGs)'s clinical and methodological characteristics and critically appraise their methodology quality.

Design We summarised the characteristics of the guidelines and recommendations and evaluated their methodological quality using the Appraisal of Guidelines Research and Evaluation II (AGREE II) instrument.

Data sources Nine databases were searched from 1 January 2010 to 20 September 2020.

Eligibility criteria for selecting studies We included the latest version of acupuncture CPGs, which must have used at least one systematic review addressing the benefits and harms of alternative care options to inform acupuncture recommendations.

Data extraction and synthesis Reviewers, working in pairs, independently screened and extracted data. When there are statistical differences among types of CPGs, we reported the data by type in the text, but when not, we reported the overall data.

Results of the 133 eligible guidelines, musculoskeletal and connective tissue diseases proved the most commonly addressed therapeutic areas. According to the AGREE II instrument, the CPG was moderate quality in the domain of clarity of scope and purpose, clarity of presentation, the rigour of development, stakeholder involvement and low quality in editorial independence, and applicability. The study identified 433 acupuncturerelated recommendations; 380 recommended the use of acupuncture, 28 recommended against the use of acupuncture and 25 considered acupuncture but did not make recommendations. Of the 303 recommendations that used Grading of Recommendations Assessment, Development and Evaluation to determine the strength of recommendations, 152 were weak recommendations, 131 were strong recommendations, of which 104 were supported by low or very low certainty evidence (discordant recommendations).

Conclusion In the past 10 years, a large number of CPGs addressing acupuncture interventions exist. Although these
Strengths and limitations of this study

- This systematic survey includes a comprehensive search of eligible clinical practice guidelines (CPGs) and systemic and explicit eligibility criteria.

- This study used the Appraisal of Guidelines Research and Evaluation II instrument to evaluate the methodological of eligible CPGs.

- This study investigated the utilisation of the Grading of Recommendations Assessment, Development and Evaluation in CPGs.

- This study documented recommendations discordant with respect to evidence strength and certainty.

- Limitations include the exclusion of guidelines not supported by any systematic reviews; our findings, therefore, do not apply to the lowest methodological quality guidelines.

guidelines may be as or more rigorous than many others, considerable room for improvement remains.

\section{INTRODUCTION}

With more than 3000 years of history, acupuncture is one of the widely used complementary and alternative therapies. ${ }^{12}$ Among the 192 WHO member states, 183 have used acupuncture. ${ }^{3}$ In Norway, $34 \%-64 \%$ of doctors recommend acupuncture to their patients. ${ }^{4}$ The increasing acupuncture application in practice has led to growing demands for clinical practice guidelines (CPGs), with many countries and academic organisations including acupuncture in their CPGs. ${ }^{56}$

Trustworthy CPGs base their recommendations on systematic reviews (SRs) assessing the benefits and harms of alternative care options, rate the certainty of the evidence and grade the strength of recommendations. ${ }^{78}$ 
CPGs help clinicians, managers and policy-makers select the best available evidence to support decision making. Despite the existing large number of acupuncture-related CPGs, the clinical and methodological status of CPGs, including recommendations, remain underexplored.

This systematic survey summarises acupuncture-related CPGs' clinical and methodological characteristics and critically assesses their methodology quality.

\section{METHODS}

\section{Definition}

\section{Acupuncture and acupuncture point}

In this study, acupuncture is used in a broad sense refers to interventions that use any stimulation on acupuncture points, including manual acupuncture, electric acupuncture (electroacupuncture), acupressure, moxibustion, warm needling, fire needling, transcutaneous electrical nerve, laser acupuncture, microsystem acupuncture, thread-embedding therapy, medicine acicula, point injection, acupoint paste; magnetic acupuncture, blood-letting therapy, acupotomy. ${ }^{9}$

Acupuncture point: the point in humans where stimulation and manipulation are performed in acupuncture therapies. ${ }^{10}$

\section{Types of CPG}

This study divides eligible CPGs into three types: acupuncture-specific CPG (all recommendations include acupuncture interventions), traditional and complementary medicine (T\&CM) CPG (all recommendations include T\&CM interventions), and Comprehensive CPG (all recommendations include conventional interventions, and some include acupuncture interventions).

\section{Acupuncture recommendation}

We define acupuncture recommendations as recommendations (including for, against or considered but did not make recommendations) in which authors considered acupuncture as a treatment or prophylactic (eg, prevent nausea and vomiting after chemotherapy) option.

\section{Conventional medicine}

Conventional medicine is defined as pharmacological, and other non-pharmacological used in conventional medicine systems to treat, prevent disease, or restore, correct, or modify physiological function. ${ }^{11}$

\section{Outcome classification}

We categorised outcomes into symptoms (eg, pain, insomnia); function (eg, poststroke motor function); surrogate outcomes (eg, blood pressure); quality of life (eg, short Form 36 survey); morality and major morbid events (eg, myocardial infarction). ${ }^{12}$

\section{Eligibility criteria}

Eligible CPGs met all the following criteria: (1) title or abstract included keywords 'CPG' or 'guideline' or 'guidelines' or 'guidance'; If the CPG had no abstract, introduction, scope, purpose, rationale, background or objectives presented at least one of these keywords; (2) full text included 'acupuncture' or 'acupressure' or 'transcutaneous electrical nerve' ; (3) the CPG used at least one SRs addressing the benefits and harms of alternative care options to inform recommendations; (4) the CPG addressed patient prevention or treatment; (5) the CPG included at least one recommendation addressing acupuncture and (6) the latest version of a CPG when multiple versions exist. This study excluded health technology assessment.

\section{Literature search}

LL, HZ and YZ developed the search strategy. The keywords included guideline, recommendation, advice, opinion, acupuncture, transcutaneous electrical nerve stimulation, acupressure, etc (detail in online supplemental material 1). Two reviewers (ZC and XS) systematically searched databases including MEDLINE, EMBASE, Trip medical database, the Guidelines International Network, Agency for Healthcare Research and Quality, National Institute for Health and Care Excellence, Canadian Medical Association's CPGs database, the New Zealand Ministry of Health, China National Knowledge Internet (CNKI), Wanfang Data, China Biology Medicine, and China Science and Technology Journal Database from 1 January 2010 to 20 September 2020. Without language restriction, we searched subject terms and free words. The reviewers managed records using EndNote (V.X9.0).

\section{CPGs selection, data extraction and analysis}

Reviewers, working in pairs, independently screened and extracted data. After title and abstract screening, reviewers retrieved and evaluated potentially eligible guidelines' full texts, resolving disagreement by discussion or consultation with a third reviewer (XS). To collect data, we designed a structured data extraction table in Excel.

Reviewers extracted two types of information from each CPG: (1) CPG level information, for example, region of the publication, types of CPG, target users, and diseases or conditions investigated, (2) recommendation level information, for example, strength and direction of the recommendation, intervention (eg, types of acupuncture and acupoints), comparators included in the recommendation, outcomes informing the recommendation.

To compare the variables across different types of CPGs, we used the $\chi^{2}$ and, when necessary, Fisher's exact test. When there are statistical differences among types of CPGs, we reported the data by type in the text, but when not, we reported the overall data. We showed all data in the tables. This study conducted the analyses using Statistical Package for the Social Sciences (SPSS, V.22) and set the significance level at $5 \%(\mathrm{p}<0.05)$. 


\section{Quality appraisal}

The study used the Appraisal of Guidelines Research and Evaluation II (AGREE-II) instrument, which includes 23 appraisal criteria (items) organised within six domains: scope and purpose, stakeholder involvement, the rigour of development, clarity of presentation, applicability, and editorial independence, to assess the quality of CPGs. Reviewers rated each item on a seven-point scale from strongly disagree (score 1) to strongly agree (score 7 ). We calculated the scaled domain score (higher score indicates better quality) according to AGREE II (the 'obtained score' was the sum of the appraisers' scores per item $)^{13}$ : (Obtained score - Minimum possible score) / (Maximum possible score - Minimum possible score). Prior to quality appraisal, eight reviewers received training in AGREE II with repeated assessment until the chance-corrected agreements were $\geq 0.6$ for each pair of reviewers in each domain. Two reviewers (ZC and XT) independently assessed each CPG, and when the discussion failed to resolve the disagreement, a third reviewer (XS) arbitrated the case.

AGREE II instrument does not set minimum domain scores or patterns of scores across domains to differentiate between high quality and low quality guidelines. As previously suggested, we considered domain and overall scores under $50 \%$ to indicate low quality, $>50 \%$ as moderate quality and $>80 \%$ as high quality. ${ }^{14-16}$

\section{Patient and public involvement}

No patient involved.

\section{RESULTS}

\section{Literature search}

Our initial search yielded 8943 citations, of which 3122 were excluded due to duplicate publication and 4848 deemed ineligible. Of the 973 full texts, we excluded 840 reports for the following reasons: 793 did not include any recommendation regarding acupuncture, 28 were not CPGs, 16 had unavailable full texts and 3 were duplicate publications. The 133 CPGs deemed eligible, including 433 acupuncture recommendations (figure 1 ).

\section{Characteristics of included CPGs and recommendations}

Table 1 and online supplemental material 2 present eligible CPGs' summary characteristics.

The organisations that conducted CPGs were from North America $(49,36.8 \%)$, Asia $(44,33.2 \%)$, Europe $(31,23.3 \%)$, Oceania $(6,4.5 \%)$ and international organisations (eg, WHO) $(3,2.2 \%)$. Guideline category proved comprehensive in $89(66.9 \%)$, acupuncture-specific CPGs in $31(23.3 \%)$ and T\&CM specific in $13(9.8 \%)$. Sixty-nine $(51.9 \%)$ used Grading of Recommendations Assessment, Development and Evaluation (GRADE) to assess the certainty of the evidence, and 59 (44.4\%) used GRADE to assess the strength of recommendation.

Figure 2 shows CPGs and recommendations' therapeutic areas distribution, quantity, and methodological

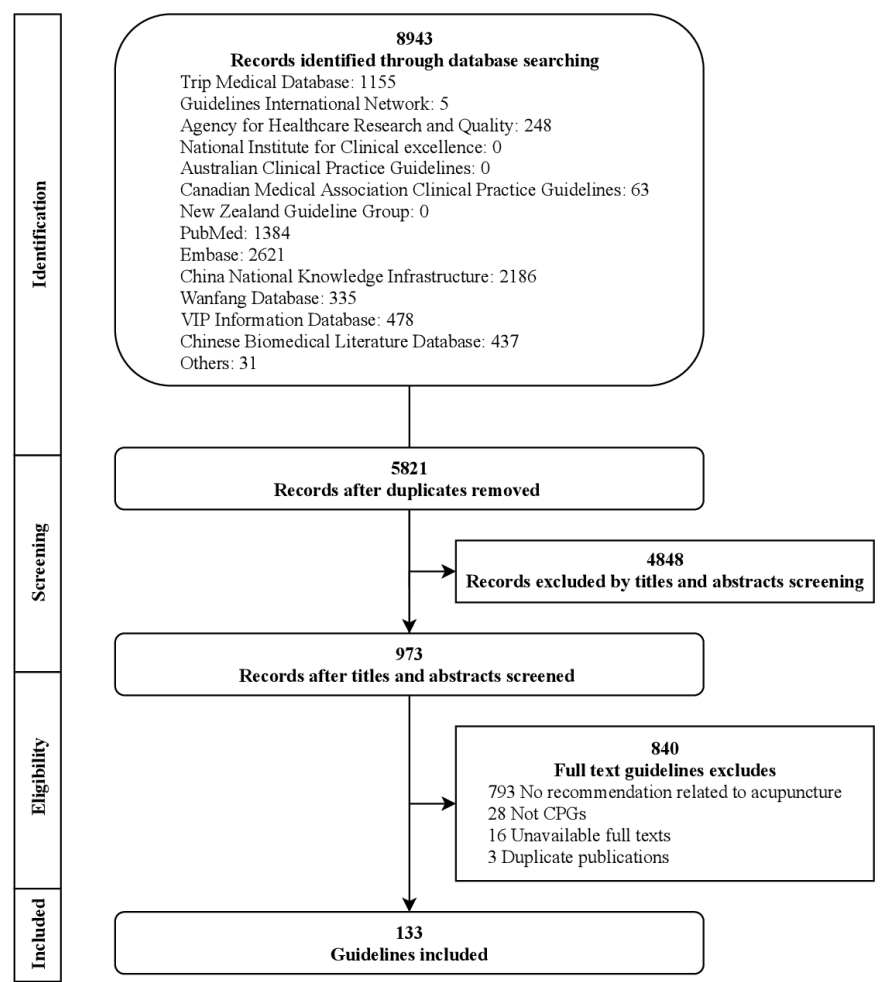

Figure 1 The Preferred Reporting Items for Systematic Reviews and Meta-Analyses flow diagram. CPG, clinical practice guideline.

rigour. Areas most frequently addressed proved musculoskeletal and connective tissue diseases $(39,29.3 \%)$, neurological disorders $(21,15.8 \%)$ and oncology (11, 8.3\%). CPGs seldom addressed endocrine, nutritional and metabolic, ophthalmology, cardiovascular diseases, respiratory diseases and dermatology (one per therapeutic area).

\section{Methodological quality of CPGs}

Table 2 presents CPGs AGREE II rating. Of all CPGs, domains evaluated by AGREE II scored from the highest to lowest include: 'scope and purpose,' 'clarity of presentation,' 'rigour of development,' 'stakeholder involvement', 'editorial independence,' and 'application.' The first four domains proved moderate quality (ie, $50 \%$ or higher rating), and the last two domains deemed low quality (ie, scored less than 50\%).

For comprehensive CPGs, 'clarity of presentation' $(75.2 \%)$, 'scope and purpose' $(74.0 \%)$, 'editorial independence (53.4\%)' and 'rigour of development' (51.6\%) proved moderate quality; meanwhile 'stakeholder involvement $(49.8 \%)$ ', and 'application (32.1\%)' proved lower quality. For T\&CM CPGs, 'clarity of presentation' $(68.2 \%)$, and 'scope and purpose' $(66.0 \%)$ were moderate quality; meanwhile 'editorial independence' (48.7\%), 'rigour of development' (45.9\%), 'stakeholder involvement' (32.7\%) and 'application' (22.4\%) were lower quality. For acupuncture-specific CPGs, 'scope and purpose' (95.2\%) was high quality; and 'clarity of presentation' (79.8\%), 'rigour of development' (71.3\%), and 'stakeholder involvement' $(67.8 \%)$ were moderate 


\begin{tabular}{|c|c|}
\hline Variable & $\mathbf{N}(\%)$ \\
\hline \multicolumn{2}{|l|}{ Region } \\
\hline International & $3(2.3)$ \\
\hline America & $49(36.8)$ \\
\hline Asia & $44(33.1)$ \\
\hline Europe & $31(23.3)$ \\
\hline Oceania & $6(4.5)$ \\
\hline \multicolumn{2}{|l|}{ Type of CPG } \\
\hline Comprehensive CPG & $89(66.9)$ \\
\hline T\&CM CPG & $13(9.8)$ \\
\hline Acupuncture-specific CPG & $31(23.3)$ \\
\hline
\end{tabular}

CPGs with at least one acupuncture recommendation supported by systematic reviews

\begin{tabular}{lc} 
Comprehensive CPG & $46(34.6)$ \\
\hline T\&CM CPG & $7(5.3)$ \\
Acupuncture-specific CPG & $12(9.0)$ \\
\hline The health intent & \\
Treatment & $129(97.0)$ \\
Prevention & $17(12.8)$
\end{tabular}

\section{Target users ${ }^{\star}$}

\begin{tabular}{lc} 
Healthcare provider & $127(95.5)$ \\
Policy-maker & $9(6.8)$ \\
Patient & $39(29.3)$ \\
Using GRADE to assess thecertainty of the evidence & $69(51.9)$ \\
Reported & $64(48.1)$ \\
Not reported & $59(44.4)$ \\
Using GRADE to assess the strength of recommendation \\
Reported & $74(55.6)$ \\
\hline
\end{tabular}

*One CPG can contribute to more than one category. CPG, clinical practice guidelines; GRADE, Grading of Recommendations Assessment, Development and Evaluation; T\&CM, traditional and complementary medicine.

quality; meanwhile 'application' (35.4\%) and 'editorial independence' $(33.2 \%)$ were lower quality.

The low rating in the 'application' domain reflects inadequate consideration of resource use $(52,39.1 \%)$ and monitoring/auditing standards. The absence of a conflict-of-interest (COI) statement $(61,45.9 \%)$ and lack of explicit explanation on how COIs are considered (58,43.6\%) from CPGs developers led to the low rating in the 'editorial independence' domain. In addition to item 5 in AGREE II, whether guideline developers considered patients' values and preferences, we evaluated whether the CPG explicitly explained how they considered it. The results showed that $43.6 \%$ reported considering patients' values and preferences, of which only less than $10.5 \%$ explicitly explained how they were considered (online supplemental 3 table A).
Acupuncture recommendations specific analyses

Evidence supporting acupuncture recommendations

Table 3 shows the evidence that supported acupuncture recommendations. Type of study design used to inform acupuncture recommendations from the most to the least frequently are randomised controlled trial and controlled clinical trial, SR and meta-analysis, observational study, narrative review, expert consensus and Chinese classic texts/textbook. Of all acupuncture recommendations, $314(72.5 \%)$ used GRADE to assess the certainty of evidence. Low or very low certainty evidence supported over $80 \%$ of the 314 acupuncture recommendations.

\section{Direction, strength and rationale of the recommendations}

Of the 433 acupuncture recommendations, 380 (87.8\%) recommended or suggested using acupuncture, 28 $(6.4 \%)$ recommended or suggested against, and 25 $(5.8 \%)$ did not make recommendations. Of the 303 ( $70.0 \%$ of all recommendations in the guidelines) recommendations that used GRADE to determine the strength of recommendations, 152 (50.2\%) were weak recommendations, and 131 (43.2\%) were strong recommendations, of which $104(79.4 \%)$ were supported by low or very low certainty evidence (we will refer to this situation as discordant recommendations ${ }^{15}$ (table 4 ).

online supplemental 3 table $\mathrm{B}$ shows the rationale when making acupuncture recommendations. For all CPGs, treatment benefits are the main reason for recommending or against acupuncture $(264,61.0 \%)$. Forty-one (36.3\%) comprehensive CPGs consider the certainty of evidence as an important issue in making recommendations, while $\mathrm{T} \& \mathrm{CM}$ and acupuncture-specific CPGs seldom consider certainty $(1,0.4 \%)$. Less than $10 \%$ of all CPGs reported patients' values and preferences, cost, adverse events and feasibility as part of their rationale for making acupuncture recommendations.

\section{Patient characteristics in acupuncture recommendations}

Acupuncture recommendations report patient information poorly. Of all acupuncture recommendations, only $16(8.7 \%)$ reported the severity of the disease. Seventeen $(15.0 \%), 3(4.5 \%)$, and 101 (39.8\%) of the comprehensive, T \& CM and acupuncture-specific CPGs reported the stage of the disease (online supplemental 3 table $\mathrm{C}$ ).

\section{Acupuncture interventions in the recommendations and remarks}

Acupuncture recommendations and remarks reported limited details regarding acupuncture intervention (table 5). Comprehensive CPGs most frequently include manual acupuncture and acupressure in acupuncture recommendations. T\&CM and acupuncture-specific CPGs most frequently include manual and electro-acupuncture in acupuncture recommendations. Most acupuncture recommendations in all CPGs focus on stand-alone usage of acupuncture therapies, and only 99 (22.9\%) recommendations use acupuncture as a combination/ adjunct therapy. Moreover, almost no recommendations reported 


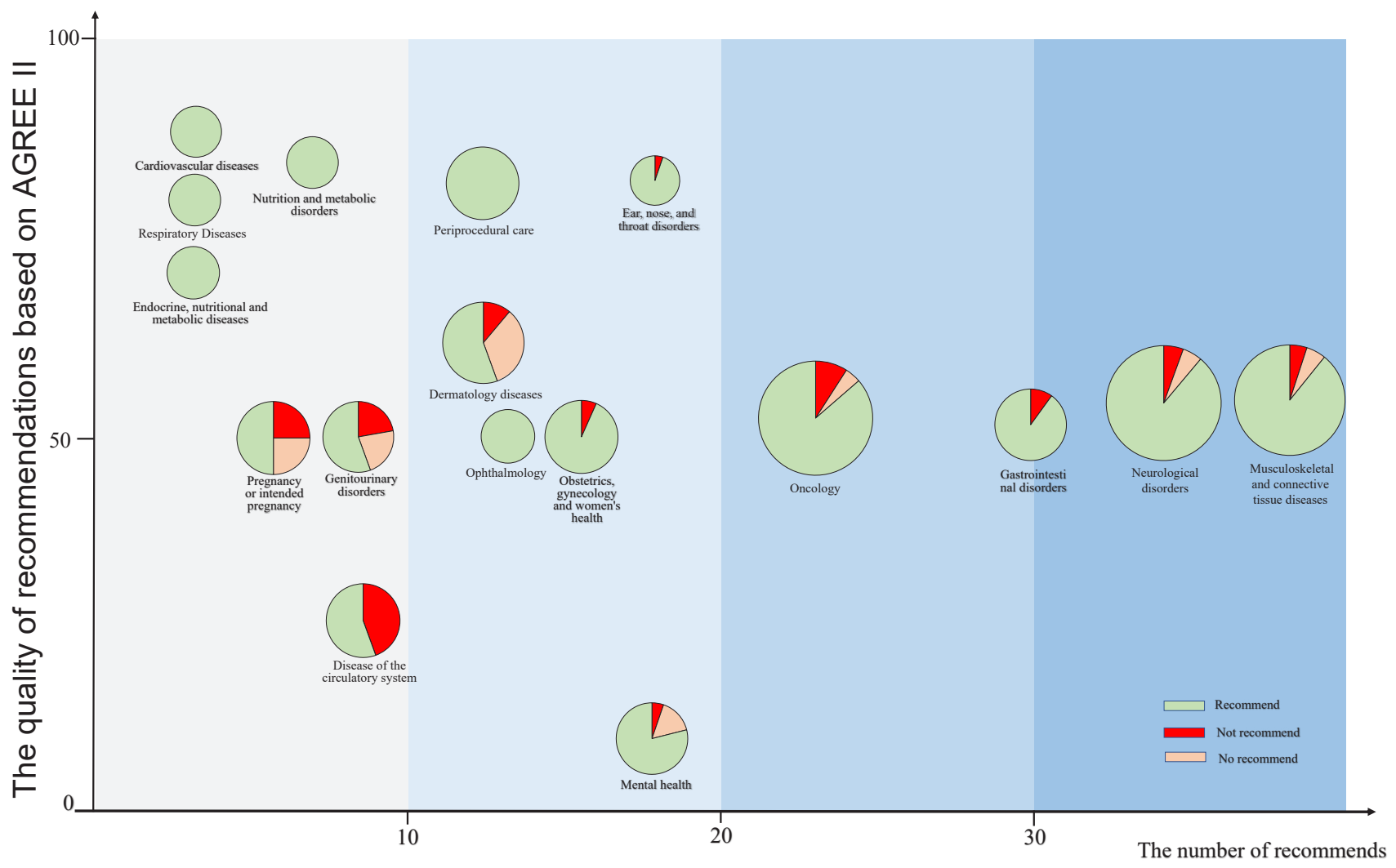

Figure 2 A multidimensional plot for acupuncture guidelines and recommendations' quantity, methodological rigour and distribution of therapeutic areas. Each pie represents a therapeutic area. (1) Pie size: number of CPGs (larger=more studies). (2) Pie colour: direction of recommendation (green: recommend for, red: recommend against; pink: did not make a recommendation). CPG, clinical practice guideline.

the acupuncture points selection except for acupuncturespecific CPGs $(206,81.1 \%)$.

\section{Comparators in acupuncture recommendations}

Approximately three-quarters of the CPGs recommendations $(323,74.6 \%)$ did not report the comparators (online supplemental 3 table D). Among all CPGs recommendations that reported the comparators, comprehensive CPGs most often compared acupuncture with no acupuncture $(12,10.6 \%)$, T\&CM CPGs most often compared acupuncture with non-pharmacological therapy (22, 33.3\%), and acupuncture-specific CPGs most often compared acupuncture with conventional medicine $(32,12.6 \%)$.

\section{Outcomes summarised to inform acupuncture recommendations}

Among all acupuncture recommendations, 130 (30.0\%) did not summarise the outcomes used to inform recommendations: $43 / 113,38 / 66$ and 49/254 were in comprehensive, T\&CM and acupuncture-specific CPGs. Of the

\begin{tabular}{|c|c|c|c|c|c|}
\hline Domain & Comprehensive CPGs & T\&CM CPGs & Acupuncture-specific CPGs & Total & $P$ value \\
\hline Scope and purpose & 74.0 & 66.0 & 95.2 & 78.4 & 0.386 \\
\hline Rigour of development & 51.6 & 45.9 & 71.3 & 56.3 & \\
\hline Clarity of presentation & 75.2 & 68.2 & 79.8 & 74.4 & \\
\hline
\end{tabular}

CPG, clinical practice guidelines; T\&CM, traditional and complementary medicine. 


\begin{tabular}{|c|c|}
\hline Variable & $\mathbf{N}(\%)$ \\
\hline \multicolumn{2}{|c|}{$\begin{array}{l}\text { Type of study supporting acupuncture } \\
\text { recommendations }{ }^{*}(n=433)\end{array}$} \\
\hline SR or meta-analysis & $135(31.2)$ \\
\hline $\mathrm{RCT}$ or $\mathrm{CCT}$ & $250(57.7)$ \\
\hline Observational studies & $114(26.3)$ \\
\hline Narrative review & $36(8.3)$ \\
\hline Expert consensus & $24(5.5)$ \\
\hline Chinese classic texts/ Textbook & $4(0.9)$ \\
\hline Not reported & $47(10.9)$ \\
\hline \multicolumn{2}{|c|}{$\begin{array}{l}\text { Recommendations used GRADE to assess thecertainty } \\
\text { of evidence }(n=314)\end{array}$} \\
\hline Comprehensive CPG & $46(40.7)$ \\
\hline T\&CM CPG & $24(36.3)$ \\
\hline Acupuncture-specific CPG & $244(96.1)$ \\
\hline
\end{tabular}

Certainty of evidencesupporting recommendations using GRADE $(n=314)$

\begin{tabular}{lc} 
High & $5(1.6)$ \\
Moderate & $33(10.5)$ \\
Low & $105(33.4)$ \\
Very low & $157(50.0)$ \\
Not reported & $14(4.5)$ \\
\hline
\end{tabular}

${ }^{*}$ One recommendation can contribute to more than one category. development, and evaluation, CCT, controlled clinical trial; CPG, clinical practice guidelines; GRADE, Grading of Recommendations Assessment, Development and Evaluation; RCT, randomised controlled trial; SR, systematic review; T\&CM, traditional and complementary medicine.

remaining 382 , from the most to the least summarised types of outcomes are: symptoms $(253,58.4 \%)$, function $(86,19.9 \%)$, quality of life $(29,6.7 \%)$, surrogate outcomes $(12,2.8 \%)$, mortality $(1,0.2 \%)$ and major morbid events $(1,0.2 \%)$ (online supplemental 3 table E).

\section{DISCUSSION}

This study identified 133 CPGs and 433 recommendations addressing acupuncture intervention published between 2010 and 2019 that most frequently addressed musculoskeletal and connective tissue diseases $(29.3 \%)$, neurological disorders (15.8\%), obstetrics, gynaecology, and women's health $(9.7 \%)$, and oncology $(9.0 \%)$ (figure 2). Guidelines overwhelmingly recommended in favour of acupuncture $(87.8 \%$ of recommendations), with the remainder split more or less evenly between recommendations against or no recommendation. Of the 303 $(70.0 \%)$ recommendations that used GRADE to determine the strength of recommendations, 152 (50.2\%) were weak recommendations, $131(43.2 \%)$ were strong recommendations, of which 104 (79.4\%) were supported by low or very low certainty evidence (discordant recommendations). According to the AGREE II instrument,
Table 4 Direction and strength of acupuncture recommendations

\begin{tabular}{lc}
\hline Variable & N (\%) \\
\hline The direction of the recommendation $(\mathbf{n = 4 3 3 )}$ & $380(87.8)$ \\
\hline In favour & $28(6.4)$ \\
\hline Against & $25(5.8)$ \\
\hline Did not make recommendations & $131(43.2)$ \\
$\begin{array}{l}\text { Strength of the recommendation assessed with GRADE } \\
\text { (n=303) }\end{array}$ & $26(19.8)$ \\
\hline Strong & $104(79.4)$ \\
\hline High and moderate certainty of the & $1(0.8)$ \\
evidence & $152(50.2)$ \\
\hline Low and very low certainty of the evidence & $10(6.6)$ \\
\hline Not reported & \\
\hline Weak & $135(88.8)$ \\
\hline High and moderate certainty of the & $7(4.6)$ \\
evidence & $20(6.6)$ \\
\hline Low and very low certainty of the evidence & \\
\hline Not reported & \\
\hline Not reported & \\
\hline
\end{tabular}

GRADE, Grading of Recommendations Assessment, Development and Evaluation.

included CPGs proved moderate quality in 'scope and purpose,' 'clarity of presentation,' 'rigour of development' and 'stakeholder involvement,' but low quality in 'editorial independence' and 'applicability' domains.

\section{Strengths and limitations}

Our study, the most comprehensive that systematically evaluated CPGs addressing acupuncture interventions, has several strengths. First, this systematic survey includes a comprehensive search of eligible CPGs, systemic and explicit application of eligibility criteria, duplicate and independent study selection and data extraction, and rigorous methodological assessment of CPGs using the AGREE-II instrument. Second, our research investigated the utilisation of the GRADE approach in CPGs and recommendations. Moreover, we documented recommendations discordant with respect to strength and certainty. Third, this survey identified the missing aspect in AGREE II that most acupuncture-related CPGs did not explicitly report how they consider patients' values and preferences, hindering guidelines' applicability.

The limitation of our study includes: (1) we did not include Japanese and South Korean guideline databases, and (2) we excluded guidelines not supported by any SRs-thus, results do not apply to the lowest methodological quality guidelines.

Relation to other studies

Jeremy $\mathrm{Y} \mathrm{Ng}$.et $a l^{17}$ investigated the quantity and quality of T\&CM (eg, herbal medicine, acupuncture, chiropractic and osteopathic manipulation) guidelines in 
Table 5 Details of acupuncture interventions in the recommendations and remarks

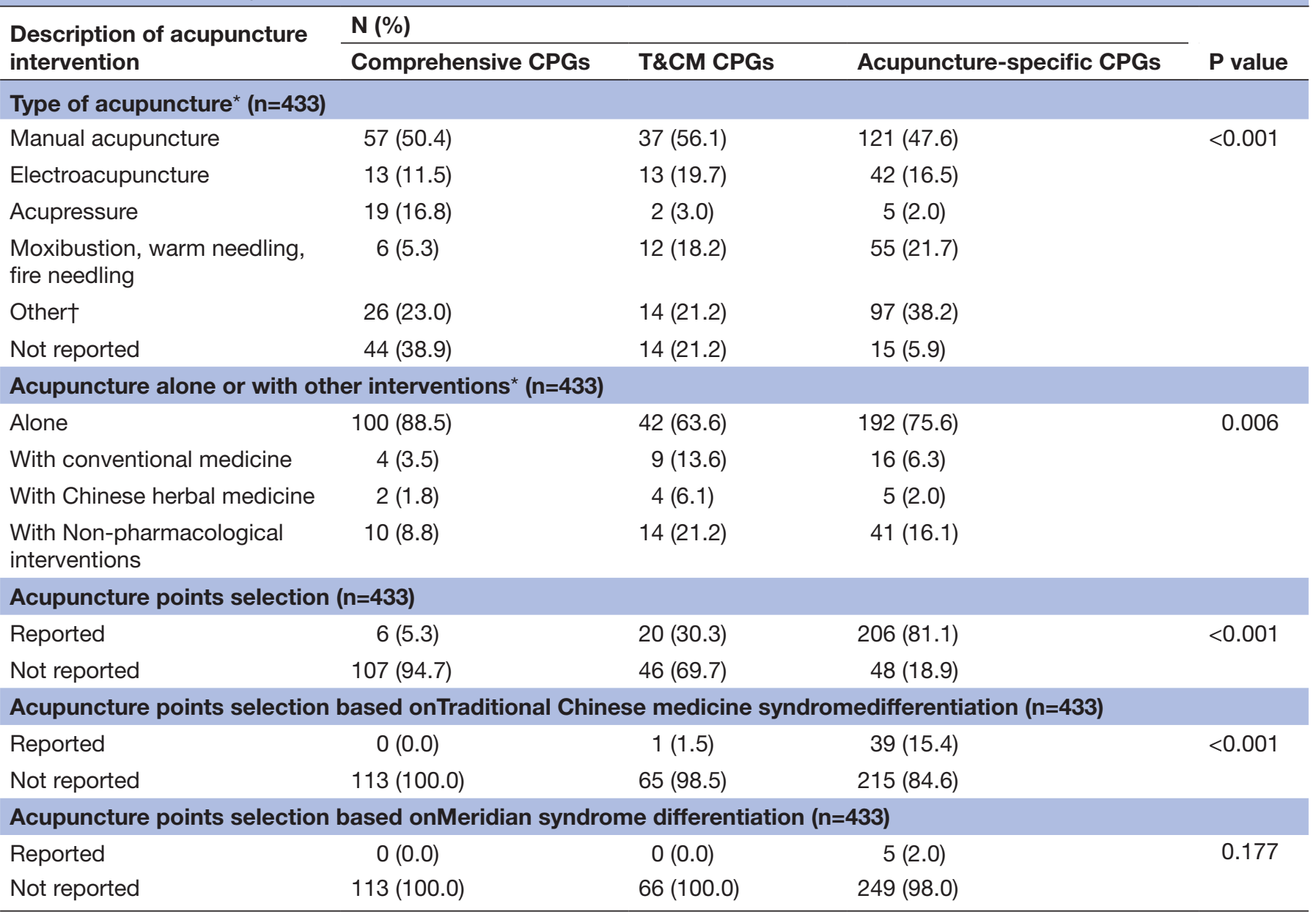

*One recommendation can contribute to more than one category.

†Include laser acupuncture, microsystem acupuncture, thread-embedding therapy, medicine acicula, point injection, transcutaneous electrical nerve, acupoint paste; magnetic acupuncture, blood-letting therapy, acupotomy.

CPG, clinical practice guidelines; T\&CM, traditional and complementary medicine.

MEDLINE, EMBASE and CINAH, etc. This survey examined a few selected interventions in $\mathrm{T} \& \mathrm{CM}$ and only included three acupuncture-related guidelines. Guo LH et $a l^{18}$ evaluated the methodological quality of acupuncture CPGs conducted by the China Institute of Acupuncture and Moxibustion. Consistent with our results, both articles identified 'applicability' and 'editorial independence' as Acupuncture-specific CPGs 'main limitations, but they omitted non-Chinese CPGs. Guo Y et at conducted a systematic survey to summarise the diseases and disorders most commonly treated with acupuncture in CPGs and concluded that acupuncture recommendations might be limited to painful conditions. They only searched the National Guideline Clearinghouse database, which might explain their failure to present the broad picture of acupuncture guidelines that our survey describes. None of the three studies analysed whether patients' values and preferences were explicitly considered, and only Wu et al summarised whether CPGs used the GRADE approach.

\section{Implications}

Acupuncture guidelines could improve by consistently supporting recommendations with SRs, explicitly considering patients' values and preferences, providing additional details regarding patients' characteristics, acupuncture interventions/definition, comparators and outcomes summarised.

Despite available evidence and the wide application of acupuncture as an adjunct therapy in practice (eg, in combination with conventional medicine) ${ }^{19-22}$ acupuncture recommendations often do not address this question. Future guidelines should much more frequently address this issue.

The GRADE approach perceives strong recommendations supported by low or very low certainty evidence (ie, discordant recommendations) as questionable and often inappropriate. ${ }^{23}$ Guidelines addressing acupuncture intervention should better understand the GRADE approach, include panels that support the GRADE approach's optimal usage, and collaborate with well-trained methodologists to avoid discordant recommendations. ${ }^{24}{ }^{25}$ Both financial and 
intellectual COIs can impact panels and guideline developers' judgement and the recommendations. ${ }^{26}{ }^{27}$ Proper management of COIs can mitigate bias related to it and increase CPGs' credibility. CPGs addressing acupuncture should report guideline' funding sources, declare and manage all guideline developers and panel members' COIs considering available literature. ${ }^{28} 29$

\section{CONCLUSION}

In the past 10 years, many CPGs addressing acupuncture interventions covering various diseases and conditions exist. Although these guidelines may be as or more rigorous than many others, considerable room for improvement remains, particularly, guideline developers should better manage COIs, explicitly considering patients values and preferences and resources utilisation, using more SRs to support recommendations and avoiding discordant recommendations.

\section{Author affiliations}

${ }^{1}$ South China Research Center for Acupuncture and Moxibustion, Medical College of Acu-Moxi and Rehabilitation, Guangzhou University of Chinese Medicine, Guangzhou, China

${ }^{2}$ Gastroenterology dept, The Third Affiliated Hospital of Beijing University of Chinese Medicine, Beijing, China

${ }^{3}$ Institute of Acupuncture and Moxibustion, China Academy of Chinese Medical Sciences, Beijing, China

${ }^{4}$ Shenzhen Luohu District Hospital of Traditional Chinese Medicine, Shenzhen, China ${ }^{5}$ Department of Acupuncture, China Academy of Chinese Medical Sciences Guang'anmen Hospital, Beijing, China

${ }^{6}$ The first Clinical College of Guangzhou University of Chinese Medicine, Guangzhou, China

${ }^{7}$ Department of Health Research Methods, Evidence, and Impact, McMaster University, Hamilton, Ontario, Canada

${ }^{8}$ Department of Medicine, Faculty of Health Sciences, McMaster University, Hamilton, Ontario, Canada

${ }^{9}$ Nottingham Ningbo GRADE center, The University of Nottingham, Ningbo, China ${ }^{10} \mathrm{CEBIM}$ (Center for Evidence Based Integrative Medicine)-Clarity Collaboration, Guang'anmen Hospital, China Academy of Chinese Medical Sciences, Beijing, China

Acknowledgements We thank Hao wen, Peiming zhang, Xiaojing Wei, Yong Jiang, Haoyu Zhang, Chen Chen, and Yiming Chen all from the Guangzhou University of Chinese Medicine, for contributing to the literature screening, data extraction and visualisation.

Contributors YQZ, LL, HZ, XJ and NX codeveloped the protocol for this systematic survey. LL, HZ and YQZ developed the search strategy. XS, ZC, YF, LL, RD, PZ, YX, $\mathrm{SC}, \mathrm{FG}$ and JF conducted the screening of studies, data extraction and critical appraisal. YQZ, XT and XS reviewed each stage of study selection and calibrated findings. XT, XS, YQZ, LL and HZ wrote the manuscript's first draft. All authors assisted in the interpretation and write-up of results. All authors approved the final manuscript prior to submission. The corresponding author attests that all listed authors meet authorship criteria and that no others have been omitted. YQZ and $X J$ are the guarantors of this paper.

Funding This study was supported by the Innovation Team and Talents Cultivation Program of the National Administration of Traditional Chinese Medicine (ZYYCXTD-C-202004); the special project of "Lingnan Modernization of Traditional Chinese Medicine" within the 2019 Guangdong Provincial Research and Development Program (2020B1111100008); the Project of First Class Universities and High-level Dual Discipline for Guangzhou University of Chinese Medicine; and the National Natural Science Foundation of China (82174527); Graduate Research Innovation Project of Guangzhou University of Chinese Medicine; The belt and road' International Cooperation Project supported by China Academy of Chinese Medical Sciences (project number: GH201901).

Disclaimer The funders had no influence on study design, data collection, analysis, decision to publish, or manuscript preparation.
Competing interests All authors have completed the ICMJE uniform disclosure form at www.icmje.org/coi_disclosure.pdf and declare funding for the submitted work as described above; no financial relationships with any organisations that might have an interest in the submitted work in the previous three years; no other relationships or activities that could appear to have influenced the submitted work.

Patient consent for publication Not applicable.

Ethics approval This study does not involve human participants.

Provenance and peer review Not commissioned; externally peer reviewed.

Data availability statement Data are available on reasonable request. The full dataset and statistical code will be available on reasonable request by emailing $\mathrm{YZ}$.

Supplemental material This content has been supplied by the author(s). It has not been vetted by BMJ Publishing Group Limited (BMJ) and may not have been peer-reviewed. Any opinions or recommendations discussed are solely those of the author(s) and are not endorsed by BMJ. BMJ disclaims all liability and responsibility arising from any reliance placed on the content. Where the content includes any translated material, BMJ does not warrant the accuracy and reliability of the translations (including but not limited to local regulations, clinical guidelines, terminology, drug names and drug dosages), and is not responsible for any error and/or omissions arising from translation and adaptation or otherwise.

Open access This is an open access article distributed in accordance with the Creative Commons Attribution Non Commercial (CC BY-NC 4.0) license, which permits others to distribute, remix, adapt, build upon this work non-commercially, and license their derivative works on different terms, provided the original work is properly cited, appropriate credit is given, any changes made indicated, and the use is non-commercial. See: http://creativecommons.org/licenses/by-nc/4.0/.

\section{ORCID iDs}

Xiaorong Tang http://orcid.org/0000-0003-4129-7544

Xiaoshuang Shi http://orcid.org/0000-0003-1515-4072

Yu-Qing Zhang http://orcid.org/0000-0002-6318-3575

\section{REFERENCES}

1 Wong JY, Rapson LM. Acupuncture in the management of pain of musculoskeletal and neurologic origin. Phys Med Rehabil Clin N Am 1999;10:531-45.

2 Ma Y, Dong M, Zhou K, et al. Publication trends in acupuncture research: a 20-year bibliometric analysis based on PubMed. PLoS One 2016;11:e0168123.

3 World Health Organization. WHO traditional medicine strategy: 2014-2023. Geneva: World Health Organization, 2013.

4 Norheim AJ, Fønnebø V. Doctors' attitudes to acupuncture--a Norwegian study. Soc Sci Med 1998;47:519-23.

5 Greenlee H, DuPont-Reyes MJ, Balneaves LG, et al. Clinical practice guidelines on the evidence-based use of integrative therapies during and after breast cancer treatment. CA Cancer J Clin 2017;67:194-232.

6 Guo Y, Zhao H, Wang F, et al. Recommendations for acupuncture in clinical practice guidelines of the National guideline clearinghouse. Chin J Integr Med 2017;23:864-70.

7 Qaseem A, Forland F, Macbeth F, et al. Guidelines international network: toward international standards for clinical practice guidelines. Ann Intern Med 2012;156:525-31.

8 Murad MH. Clinical practice guidelines: a primer on development and dissemination. Mayo Clin Proc 2017;92:423-33.

9 World Health Organization. Acupuncture: review and analysis of reports on controlled clinical trials. Geneva: World Health Organization, 2002.

10 World Health Organization. World Health organization. Regional office for the Western Pacific. WHO international standard terminologies on traditional medicine in the Western Pacific region. WHO Regional Office for the Western Pacific, 2007.

11 WHO. WHO global report on traditional and complementary medicine 2019. Geneva: World Health Organization, 2019.

12 Evidence mapping and overview of systematic reviews of the effects of acupuncture therapies [Article paper of collection - not yet published].

13 Brouwers MC, Kho ME, Browman GP, et al. Agree Il: advancing Guideline development, reporting, and evaluation in health care. Prev Med 2010;51:421-4.

14 Andrade R, Pereira R, van Cingel R, et al. How should clinicians rehabilitate patients after ACL reconstruction? A systematic review of clinical practice guidelines (CpGs) with a focus on quality appraisal (agree II). Br J Sports Med 2020;54:512-9. 
15 Chiappini E, Bortone B, Galli L, et al. Guidelines for the symptomatic management of fever in children: systematic review of the literature and quality appraisal with agree II. BMJ Open 2017;7:e015404.

16 Isaac A, Saginur M, Hartling L, et al. Quality of reporting and evidence in American Academy of pediatrics guidelines. Pediatrics 2013;131:732-8.

$17 \mathrm{Ng}$ JY, Liang L, Gagliardi AR. The quantity and quality of complementary and alternative medicine clinical practice guidelines on herbal medicines, acupuncture and spinal manipulation: systematic review and assessment using agree II. BMC Complement Altern Med 2016;16:425.

18 Guo L-H, Ma Y, Wu X-D. [AGREE-based evaluation and content analysis of evidence-based clinical practice guidelines for acupuncture-moxibustion]. Zhongguo Zhen Jiu 2019;39:1223-8.

19 Zhao L, Li D, Zheng $\mathrm{H}$, et al. Acupuncture as adjunctive therapy for chronic stable angina: a randomized clinical trial. JAMA Intern Med 2019;179:1388-97.

20 Jang S, Ko Y, Sasaki Y, et al. Acupuncture as an adjuvant therapy for management of treatment-related symptoms in breast cancer patients: systematic review and meta-analysis (PRISMA-compliant). Medicine 2020;99:e21820.

21 Fogarty S, Harris D, Zaslawski C, et al. Acupuncture as an adjunct therapy in the treatment of eating disorders: a randomised crossover pilot study. Complement Ther Med 2010;18:233-40.

22 Whitehurst DGT, Bryan S, Hay EM, et al. Cost-Effectiveness of acupuncture care as an adjunct to exercise-based physical therapy for osteoarthritis of the knee. Phys Ther 2011;91:630-41.
23 Agoritsas T, Merglen A, Heen AF, et al. UpToDate adherence to grade criteria for strong recommendations: an analytical survey. BMJ Open 2017;7:e018593.

24 Andrews JC, Schünemann HJ, Oxman AD, et al. Grade guidelines: 15. going from evidence to recommendation-determinants of a recommendation's direction and strength. J Clin Epidemiol 2013;66:726-35.

25 Alexander PE, Gionfriddo MR, Li S-A, et al. A number of factors explain why who guideline developers make strong recommendations inconsistent with grade guidance. J Clin Epidemiol 2016;70:111-22.

26 George JN, Vesely SK, Woolf SH. Conflicts of interest and clinical recommendations: comparison of two concurrent clinical practice guidelines for primary immune thrombocytopenia developed by different methods. Am J Med Qual 2014;29:53-60.

27 Grundy Q, Mayes C, Holloway K, et al. Conflict of interest as ethical shorthand: understanding the range and nature of "non-financial conflict of interest" in biomedicine. J Clin Epidemiol 2020;120:1-7.

28 Bou-Karroum L, Hakoum MB, Hammoud MZ, et al. Reporting of financial and Non-financial conflicts of interest in systematic reviews on health policy and systems research: a cross sectional survey. Int $J$ Health Policy Manag 2018;7:711-7.

29 Hakoum MB, Bou-Karroum L, Al-Gibbawi M, et al. Reporting of conflicts of interest by authors of primary studies on health policy and systems research: a cross-sectional survey. BMJ Open 2020;10:e032425. 